\title{
Organically bound tritium (OBT) behaviour and analysis: outcomes of the seminar held in Balaruc-les-Bains in May 2012
}

N. BAGLAN ${ }^{1}$, S.B. KIM ${ }^{2}$, C. COSSONNET $^{3}$, I.W. CROUDACE ${ }^{4}$, M. FOURNIER ${ }^{5}$, D. GALERIU ${ }^{6}$, P.E. WARWICK ${ }^{4}$, N. MOMOSHIMA ${ }^{7}$, E. ANSOBORLO ${ }^{8}$

(Manuscript received 22 October 2012, accepted 22 November 2012)

ABSTRACT Within the last ten years there has been increasing interest in tritium behaviour and distribution in the environment. This is based partly on empirical findings that tritium discharged mostly as HTO can become transformed into organic forms in environmental samples. An international workshop was convened in France in 2012 to gather the scientific community interested in organically bound tritium to share their experience and to establish a current state of knowledge. This paper summarises the outcome of the workshop, which aimed to improve skills concerning $O B T$ (Organically Bound Tritium) determination, transfer and behaviour in the environment. In order to improve $O B T$ measurement credibility, it was decided to conduct and promote $O B T$ analysis through inter-laboratory exercises. This practice will reduce uncertainty in $O B T$ analysis results, providing better $O B T$ model validation data and public dose assessments.

Keywords: tritium / organically bound tritium / international standpoint / inter-laboratory exercises

\section{Introduction}

In most countries where tritium releases to the environment occur, there is increasing interest in tritium's behaviour and its potential impact for several reasons (AGIR, 2007; CNSC, 2010; ASN, 2010):

- ITER, the new experimental reactor with high tritium throughput, might be in operation by 2025 ,

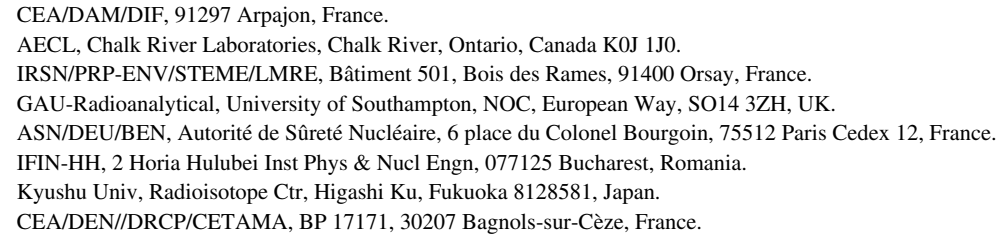


- new PWR nuclear reactors (EPR) characterised by higher tritium discharge levels are under construction or planned in several countries (China, Finland, France, UK),

- current heavy water reactors with high tritium load which are characterised by higher tritium discharge than PWR (Canada, China, Korea and Romania),

- the increasing tritium will not only be found as tritiated water but would become incorporated into environmental organic compounds, and there are questions about the impact of the various chemical species of tritium on biological systems,

- its high energy deposition along its short track length (LET) is of interest.

For most of these reasons interest in $O B T$ has grown over the last decade, with a developing focus on its analysis and environmental behaviour. Analytically, the measurement of $O B T$ concentrations in environmental samples is a longer and more complex procedure than the measurement of HTO, and has a higher associated uncertainty.

Therefore, the first international workshop dealing with $O B T$ as a central thematic topic was held in the south of France from May 22-24, 2012. The aim of this workshop was to establish current knowledge on tritium speciation and analysis, and to create an international working group (WG) dedicated to $O B T$ analysis.

\section{Current situation}

Hydrogen, the simplest and most common element, has three isotopes:

- protium $\left({ }^{1} \mathrm{H}\right)$, which contains only a proton in its nucleus and is by far the most common hydrogen isotope,

- deuterium $\left({ }^{2} \mathrm{H}\right.$ or $\left.\mathrm{D}\right)$, which contains a proton and a neutron

- tritium $\left({ }^{3} \mathrm{H}\right.$ or T), which has a proton and two neutrons, and is radioactive.

Tritium is produced naturally through various interactions of cosmic rays and atoms in the atmosphere, but is also a by-product of the operation of nuclear and research reactors.

Tritium decays to a stable helium-3 by emitting an electron from its nucleus (beta radiation) and an antineutrino. It has a radioactive half-life of $12.312 \pm$ 0.025 years (LNE, 2006). The emitted beta particle has a mean energy of $5.7 \mathrm{keV}$ and a maximum energy of $18.6 \mathrm{keV}$. Due to its very low energy, tritium's beta radiation has a very short range (approximately $6 \mu \mathrm{m}$ ) in dry air, is completely absorbed by sheets of plastic, glass or metal, and cannot penetrate dead layers of skin. However, this beta radiation can pose a health risk if tritium is taken into the body because despite this very low energy, these particles possess sufficient energy to ionise atoms and molecules on the cellular scale. 
Radioactive releases to the environment have significantly decreased over the last few decades from civil nuclear facilities, with the exception of tritium and ${ }^{85} \mathrm{Kr}$. Discharges of this radionuclide are forecast to increase due to expected changes in the fuel management methods used by Nuclear Power Plants (NPPs), to new tritium-emitting facilities, including planned power plants, and the ITER project.

Tritium can exist in several forms, such as gaseous (HT, HTO, organic molecules), liquid (HTO or organic molecules in solution) or $O B T$, which can become incorporated into living organisms (vegetables, animals, humans). Given these complexities and the probability of increasing concentrations, it is necessary to be able to accurately determine the various species of tritium in the environment for public and regulatory assurance.

Following from improvements in analytical data it will be possible to evaluate the behaviour of tritium in the environment, in particular focusing on potential accumulation of $O B T$ in organisms and for assessing the biological impact of tritium in humans. However, even in cases where tritium monitoring of atmospheric and liquid releases is currently carried out, very few countries are measuring $O B T$ despite the fact that its analysis might become a part of regular monitoring in the next few years. Indeed, the French nuclear authority (ASN) estimates that tritium monitoring in the environment and throughout the food chain, with recognised methods, must be increased. Therefore, the levels of practice are completely different in countries where $O B T$ activity is determined within research or impact assessment projects (Canada, France, Japan, UK) compared with those where $O B T$ analysis has just become operational (Korea, Romania, Russia, Switzerland). In addition, $O B T$ analysis could be difficult or tedious to handle, implying that very few data are available when they are needed to validate models to make the public more confident with predictions. Moreover, there are currently no Certified Reference Materials (CRM) and no measurement standards set by the International Organization for Standardization (ISO) relating to $O B T$. Indeed, there is only one validated procedure that was set up by the French Commission for the Establishment of Analytical Methods (CETAMA) (method 384) through inter-laboratory exercises. In the UK an informal intercomparison was established by the Health Protection Agency that investigated sewage sludge generated by a Welsh wastewater treatment works and more recently, samples of this have been included in an NPL Programme (2012 Intercomparison Exercise).

A clear conclusion from the current $O B T$ workshop showed that different procedures were used by different laboratories participating in intercomparisons organised in France and Canada. The current potential for variations in analytical data could lead to erroneous dose assessments. Thus, it is necessary to validate the 
analytical procedures and to establish robust international standards. For these reasons, the CEA (Commissariat à l'énergie Atomique) and AECL (Atomic Energy Canada Limited) organised a workshop to bring together experts from Canada, Asia and Europe with broad interests in tritium (i.e. modelling, analysis, health physics, etc.) to debate environmental tritium behaviour (analysis, speciation, migration, etc.) and to establish an international working group dedicated to $O B T$ and its analysis.

\section{The workshop}

The 1st workshop on $O B T$ and its analysis was initially organised through established links and collaborations between the CEA and AECL. The workshop took place in Balaruc les Bains (France), near Montpellier from May 22-24, 2012. Forty-five scientists from nine countries (Austria, Canada, France, Germany, Korea, Romania, Russia, Switzerland and the United Kingdom) participated in the workshop, giving fifteen presentations.

\subsection{Environmental monitoring}

During the workshop, the presentations dealt with several aspects of tritium measurement in a broad variety of samples within several matrices: environmental, but also concrete and other materials associated with nuclear decommissioning.

\subsubsection{OBT in soil depths around Chalk River Laboratories (S.B. Kim and M. Bredlaw)}

Atomic Energy of Canada Limited (AECL)'s Chalk River Laboratories (CRL) is a large nuclear research and test establishment with nuclear and non-nuclear facilities located in Chalk River, Ontario. This study was conducted to consolidate the environmental effects at CRL following a continuous atmospheric tritium release. Soil samples were collected at depths of up to $20 \mathrm{~cm}$ using soil probes at the CRL site and surrounding areas. The samples were sectioned at 5-cm intervals, and $H T O$ and $O B T$ concentrations were measured in the samples. The HTO concentration was the highest in surface soil water and plant leaves at a given sampling point. This result suggests that surface soil water and tissue free water tritium in plants significantly reflect the surrounding atmospheric tritium concentration. The top layer of soil generally had the highest $O B T$ concentration among collected soil samples. The results suggest that $O B T$ concentrations are different compared with HTO concentrations at the same site and can be representative of the previously released environmental tritium at the sampling point. The relationship between the $O B T$ concentration in soil and the tritium released to the environment will be useful information to evaluate environmental tritium effects and the fate of tritium in the terrestrial ecosystem. 


\subsubsection{Tritium in the vicinity of a continuous atmospheric release. From environment to Man (P. Le Goff, L. Vichot and P. Guétat)}

Tritium is mainly released to the environment as $H T O$ or $H T$. $H T$ transfers are slow between the different environmental compartments and its oxidation to $H T O$, in particular by soil microorganisms, is its main fate. HTO is omnipresent in abiotic or biotic compartments. By fast exchange, HTO and labile organic hydrogen achieve equilibrium.

Plants, through photosynthesis, certainly synthesise more $N E-O B T$ (nonexchangeable $O B T$ ) from $H T O$ than animals or other beings. Animals, by consumption of tritiated water and foods, integrate tritium in their tissues and fluids. The incorporation as water or organic molecules depends on the form of the tritium ingested. Some experiments have shown that animals synthesise $N E-O B T$ from $H T O$, although it represents a small fraction (about $1 \%$ of the activity ingested). When animals incorporate tritiated organic compounds, the absorption rate in their own organic compounds and retention time are higher than for HTO incorporation.

However, $O B T$ is not non-exchangeable organic tritium and includes many molecules with different kinetics and functions. This is why it seems necessary to know how $O B T$ is composed: at least what parts of proteins, carbohydrates and lipids? Differences in the metabolism of each of these kinds of molecules certainly influence the amount and concentration in an organism, along a food chain and throughout an ecosystem.

\subsubsection{Tritium in the freshwater system of the Yenisei river (L. Bondareva)}

Located on the right bank of the river Yenisei is the Mining and Chemical Combine (MCC), Rosatom (Zheleznogorsk). The operation of this enterprise resulted in a significant amount of technogenic radionuclides, including tritium, being emitted into the environment, which potentially entered the Yenisei river ecosystem.

During 2000-2011 monitoring was carried out to estimate the tritium content in the floodplain of the river Yenisei, in the zone from the city of Krasnoyarsk to the town of Igarka (1760 km downstream). The tritium content in the water of the Yenisei river does not exceed the background level for the area under investigation almost in the entire zone, being within the range from 4 to $6 \mathrm{~Bq} \cdot \mathrm{L}^{-1}$. The discharge of tritium into the Kara Sea correlates with the global values of the tritium content in water sources of the northern hemisphere. However, in the impact zone of Mining and Chemical Combine (MCC) as well as in one of the right tributaries of 
the Yenisei, the river Bolshaya Tel, the tritium content considerably exceeds the background level, with observed concentrations up to 200 and 68 Bq.L ${ }^{-1}$ for the river Yenisei and Bolshaya Tel, respectively.

When studying the tritium interaction with bottom sediments and floodplain soils in the entire area of the Yenisei (from the city of Krasnoyarsk to the Strelka settlement) it was found that the tritium content is not more than $\sim 4 \mathrm{~Bq} . \mathrm{L}^{-1}$. This content is taken to be the background level for the area under study. However, when analysing the samples of the pore water of bottom sediments and floodplain soils in the river Bolshaya Tel, tritium content exceeding background values (up to $23 \mathrm{~Bq} \cdot \mathrm{kg}^{-1}$ ) as well as the presence of the radionuclide ${ }^{14} \mathrm{C}$ (up to $\sim 6 \mathrm{~Bq} \cdot \mathrm{kg}^{-1}$ ) were found.

The conducted experiments on tritium retention by different soils showed that tritium is slightly retained by geological rocks, with a retention level depending on the content of organic matter and clay minerals with which tritium combines into non-exchangeable forms.

The evidence obtained for the aquatic and air-aquatic plants revealed that the tritium content in the extracted water of the wet weight of the plants for the background area of the river Yenisei (upstream of the MCC discharge) varies from 4 to $7 \mathrm{~Bq} \cdot \mathrm{L}^{-1}$, which is, first of all, due to the physiological features of the plant species studied and, consequently, due to the pore water content. Near the MCC reactor water discharge the tritium content in plants and are ten times higher than the background level (51 Bq. $\mathrm{kg}^{-1}$ wet weight). Estimation of the tritium contribution in the form of $O B T$ revealed that up to $15 \%$ of the total tritium can be in the form of $O B T$, which transforms from $H T O$ resulting from the photosynthesis of living organisms.

\subsubsection{Tritium concentration in different surface waters (C. Varlam, I. Stefanescu, A. Soare and I. Faurescu)}

The atmospheric testing of fusion weapons increased the inventory of tritium on the Earth's surface, in the early ' 60 s, by more than two orders of magnitude. Since the maximum of the ${ }^{3} \mathrm{H}$ bomb, the ${ }^{3} \mathrm{H}$ content in precipitation has decreased, approaching natural levels nowadays. Another tritium source, besides natural production, is anthropogenic activities such as nuclear power plants, the watch industry, consumer products and medical wastes.

The nuclear activity developed along the Danube is present in the recorded values at the maximum values of $66.7 \pm 0.5 \mathrm{TU}$ (one tritium unit $=0.118 \mathrm{~Bq} \cdot \mathrm{L}^{-1}$ ) (December 2002), and $129 \pm 0.5 \mathrm{TU}$ (July 2004). The tritium concentration in the Danube's water has decreased in the last few years despite the numerous nuclear 
power plants settled on its basin. The Danube river lies west from the Black sea in Central and South-eastern Europe. Due to its geological and geographical conditions, the Danube river basin can be divided into 3 main parts: the upper Danube basin, middle Danube basin and lower Danube basin. 65\% of the lower Danube basin is the natural border between Romania and Bulgaria. Two important nuclear plants for both countries are found in this region: Kozloduy NPP and Cernavoda NPP. Taking into account the future development of the important Romanian nuclear objective, knowledge of the present status of the tritium level is a necessity.

Therefore, an extensive monitoring programme for this isotope, along the Romanian sector of the Danube river basin, starting with Cazane Gorge and ending with the three branches of the Danube Delta, was started. The different tributaries from this sector, Cerna, Jiu, Olt and Arges, were also included in the monitoring programme.

\subsubsection{Environmental monitoring of the tritium level in the upper Rhône river (C. Antonelli, S. Estier, C. Cossonnet and P. Jean-Baptiste)}

Between 2001 and 2004 the IRSN undertook several field campaigns, in order to study tritium levels in the aquatic system of the Rhône river, from Geneva lake to Caderousse, i.e. downstream from the last nuclear site implanted on the river. Suspended and deposited sediments, aquatic plants and fish were sampled for $H T O$ and $O B T$ determination. The results underlined high levels of $O B T$ in suspended sediments and deposited sediments, with a decreasing trend downstream from the upper part of the Rhône river. This study concluded that $O B T$ in the upper Rhône river was linked to the mobilisation of ancient sediments coming from the banks of the river which were contaminated by tritium originating from the upper part of the Rhône river catchment, probably in relation to both the French and Swiss watch industries.

Therefore, the IRSN and OFSP (Office fédéral de la santé publique) decided to organise a common measurement campaign. During summer 2009, fish, aquatic plants and fine sediments were sampled, focusing on sites located near dumps or waste incineration plants as well as near tributary confluences with the Rhône river. The results of the 2009 campaign underline a global decrease in the tritium levels measured in fluvial sediments in comparison with the previous ones acquired between 2001 and 2004. The mean activity recorded in 2009 was about 4 times lower that those observed in the past. This can be explained by, on one hand, the sedimentary dynamic of the Rhône river, which was affected by floods that could have diluted the signal over a larger area and, on the other hand, by the complete cessation meanwhile of the use of tritiated luminous painting in the 
Rhône basin in Switzerland. The results did not allow identifying significant discharge sources of tritium along the river.

\subsubsection{Sampling by bubbling of atmospheric tritium in organic forms (L. Tenailleau, M. Mahu, G. Dougniaux, M. Calvez and M. Piccione)}

Tritium is present in the small nuclear diffuse, such as night vision supplies or watches, incorporated in paint. This $O B T$ is exchangeable in a non-negligible amount which could be measured through air moisture analysis close to radioluminescent items.

The whole transfer process from $E-O B T$ to the measured tritium in both $H T O$ (tritiated water) and VOT (Volatile Organic Tritium) forms is not yet fully understood. The distinction has to be made because of the impact of these two types of compounds on the human body: the ingestion dose coefficient for OBT $\left(4.2 \times 10^{-11} \mathrm{~Sv} / \mathrm{Bq}\right)$ is almost three times higher than the HTO dose coefficient $\left(1.8 \times 10^{-11} \mathrm{~Sv} / \mathrm{Bq}\right)($ ICRP, 1995).

To measure the emissions related to radioluminescent items, a bubbling system (MARC7000: BS) coupled to a liquid scintillation counter (TriCarb: LSC) was used.

The origin of the VOT measured has to be found as it might come from a direct degassing of the radioluminescent paint, or the tritium might combine with some VOCs (Volatile Organic compounds) present in the air.

We inserted an item into a one-metre-cube tank and regularly took air samples with the bubbling system $(50 \mathrm{~L} / \mathrm{h}$ flow rate). The VOT/HTO ratio was always under $1 \%$, which means the tritium is not degassed from the paint directly as $H T$ or VOT.

Indeed, VOT is directly linked to VOCs present in air, and is trapped by dissolution in the last two vials of the bubbling system. The next point to consider will be an estimation of the VOT/HTO ratio, depending on the humidity rate and concentration of $V O C$ in the air.

\subsubsection{Evidence for the preservation of $O B T$ in intertidal and subtidal sediments of the Severn estuary (I.W. Croudace and P.E. Warwick)}

The macrotidal Severn estuary (south-western UK) has received a broad range of industrial discharges since the beginning of the Industrial Revolution. A more recent anthropogenic input to the estuary from 1980-2008 was organically bound tritium, $O B T$, derived from a specialised industrial laboratory producing custom radiolabelled compounds for life science research and diagnostic testing. While it 
was generally acknowledged that the radiological impact of the tritium discharges into the Estuary was small, public concern motivated the company and regulatory agencies to commission several research studies from 1998-2005 to understand the environmental impact of the $O B T$ discharges better (Croudace et al., 2012).

Two key observations made were that the $O B T$ compounds were strongly bound to the clay/silt fraction of sediment and that the down-core $O B T$ profiles in inter-tidal and sub-tidal sediments were broadly similar to the discharge record. Geochemical and chronometric methods $\left(\mathrm{Cu}, \mathrm{Pb}\right.$ and $\mathrm{Zn}$ elemental profiles, ${ }^{210} \mathrm{~Pb}$, ${ }^{137} \mathrm{Cs}$ ) provided corroboration of the $O B T$ record. A key additional piece of evidence that firmly authenticates the established chronologies was the discovery of a previously unreported sedimentary marker layer that was generated by a major storm surge that occurred on 13th December, 1981. This extreme event caused significant erosion of the estuarine sediment and acts as a clear time marker in the sedimentary record which coincidentally immediately precedes the $O B T$ discharges. The Severn $O B T$ study provides clear evidence of systematic accumulation of $O B T$ in specific sedimentary sinks, probably mediated in part by the presence of anthropogenic carbonaceous sorbents. An estimation of the $O B T$ depositional inventory shows it represents only a very small fraction of the total discharge. The relatively modest retention in the Severn system reflects the particular dynamics of this highly macrotidal, sediment-starved estuary.

\section{Short conclusion}

As a consequence of its versatility, tritium is present in a wide range of samples and in various forms, including HT, HTO and OBT, but also in organic molecules. Therefore, to separate all contributions its analysis is demanding and requires a consistent approach to avoid misunderstandings. For example, often both the tritium incorporated by living organisms and in organic molecules is called OBT. Therefore, it is timely to provide clear definitions on the tritium form to avoid any misunderstandings among the various communities.

\subsection{Tritium sources and modelling}

A close correlation between tritium emission and content in plants is observed. However, uncertainties about tritium compartmentation within plants (translocation, fractionation, influence of the type of tritium) remain very important. It follows that the public does not really trust the results being produced from the models and want to see more experimental data. 


\subsubsection{Distribution and evolution of Organically Bound Tritium in freshwater ecosystems in the environment of nuclear power plants in France (F. Siclet and G. Gontier)}

Measurements of organically bound tritium in the aquatic environments of French NPPs started in 1977. Since then, more than 600 measurements have been performed periodically in fish, vegetation and sediments, upstream and downstream of the $15 \mathrm{NPP}$ sites located along rivers. Examination of the results shows that the influence of PWR tritium discharges is detectable only in rivers, with low background $O B T$ activities. In these situations, the observed increase in plant and fish $O B T$ is proportional to the discharge-added $H T O$ concentration in water, in agreement with specific activity models. No influence of NPP liquid discharges on the tritium level in sediment has been observed.

Upstream of all NPPs, $O B T$ levels in sediments are always higher than in plants and fish, themselves higher than $H T O$ in river water. The magnitude of these differences and the long-term trends depend on the river basin and can be explained by the varying nature of tritium sources. In river catchments, where atmospheric test fallout is the main source of tritium, a decreasing trend in $O B T$ levels is observed over the last thirty years, the $O B T$ level in sediment still being higher than $O B T$ in fish and plants by a factor of 10. In the Rhône and Rhine river basins, an additional source of tritium, probably originating from the luminous paint industry, increases the $O B T$ levels in sediment up to several thousands of Bq. $\mathrm{L}^{-1}$ with no long-term change; concentrations in plants and fish are lower by 3 orders of magnitude.

\subsubsection{Open problems in OBT modelling in crops (A. Melintescu and D. Galeriu)}

Most of the existing models developed for tritium dynamics in crops are limited in their details of $O B T$ formation in crops during the daytime and they completely skip the $O B T$ formation during the night-time.

During the daytime, photosynthates are produced, exported and accumulated in the leaves. Tritiated water $(H T O)$, if it is present in leaves, enters straight into the photosynthates as an impurity and in respect to water $\left(\mathrm{H}_{2} \mathrm{O}\right)$, there is a discrimination factor. All the subsequent processes where carbohydrates, proteins and lipids are produced follow the same metabolic pathway as the bound hydrogen.

$O B T$ production during the night-time needs much detailed understanding of the plant processes, because no assimilate is produced in the night. In photosynthesis, soluble sugars and insoluble starch are produced. Starch is accumulated in leaves during the daytime and in night conditions, starch is 
transformed (hydrolysed) into soluble sugars and consequently used in plant metabolism. Starch hydrolysis in the presence of $H T O$ is not enough in order to explain the $O B T$ formation at night. Many biochemical processes are common for both growth and maintenance respiration and among them the protein turnover can contribute to $O B T$ production. $O B T$ formation at night for adult leaves can be explained by the protein turnover. For the other plant parts and mostly for the edible plant parts, more processes must be considered in order to model the $O B T$ formation at night without calibration with experiments.

To quantify all the processes involved in $O B T$ production at night, much experimental effort is still needed together with a better understanding of the recent progresses in plant biochemical research. Work dedicated to the subject is ongoing and the results will be provided in the near future.

\subsubsection{Briefing of experimental knowledge of $O B T$ in plants (D. Galeriu and A. Melintescu)}

The assessment of the tritium ingestion dose after routine or accidental emissions requires the tritiated water (HTO) concentration in food, but the $O B T$ concentration also has a large contribution. For practical reasons, it is important to know the $O B T$ concentration dynamics in plants until harvest and to accomplish this task, both measurements and modelling trials are important. In the last ten years, many experiments have been performed for a few key crops in various countries, without any internationally agreed protocol for $O B T$ measurements. Considering this limited aspect, published and unpublished experimental data were collected and analysed. For a common view of data analysis, the translocation index $(T L I)$ was used. The $T L I$ represents the ratio between the concentration of the combustion water at harvest and the concentration of $H T O$ in leaves at the end of exposure.

A revision of the experimental data was performed in order to reveal the difference between $O B T$ formation during the daytime and night-time. There are experimental facts which demonstrate that $O B T$ production at night is significant and it could have a large impact on the ingestion dose.

For infants, it is known that the critical pathway for $O B T$ ingestion is air pasture - milk, but experimental data for $O B T$ dynamics in pastures are lacking. In future, this must be a priority for experimental work.

\section{Short conclusion}

Research is necessary to have a better insight into tritium behaviour in the environment after discharge and also for its migration to man. For that, both 
experimental data and models are required. However, to validate models and to increase the confidence in the results obtained by using them, accurate experimental tritium data that could also be more specific to plant compartments and fractions are necessary.

\subsection{Analysis}

There are many different methods for analysing the various forms of tritium in environmental samples; however, there are no published standard methods for measuring $O B T$. Furthermore, there are no certified reference materials of $O B T$ for environmental samples. As a general feature, the measurement of the various tritium fractions requires several stages, potentially leading to the use of different analytical procedures and apparatuses to treat samples. Moreover, the distinction between exchangeable $O B T$ (atoms bound to oxygen and nitrogen; $E-O B T$ ) and non-exchangeable $O B T$ (atoms bound to carbon; $N E-O B T$ ) is difficult to observe due to the difference in solubility of the organic molecules forming the environmental samples. As a result, different behaviours are observed during the labile exchange stage where, in addition to the isotopic exchange between the sample and non-tritiated water, some organic molecules are solubilised. Moreover, for $O B T$ there is no consensus on the unit to report the activity values. Indeed, results are given either in Bq. $\mathrm{L}^{-1}$ of combustion water, $\mathrm{Bq} \cdot \mathrm{kg}^{-1}$ of dry sample or Bq. $\mathrm{kg}^{-1}$ of fresh sample without any other information. This can make any comparison of the results somewhat problematic. When tritium fractions have to be analysed, the sampling and storage are key points, and caution is necessary to avoid any uncontrolled variation.

\subsubsection{Development of robust strategies for sampling, storage and analysis of ${ }^{3} \mathrm{H}$-contaminated materials as part of decommissioning waste characterisation (P.E. Warwick, I.W. Croudace, D. Kim and J. Oh)}

Tritium is routinely encountered in a diverse range of matrices during decommissioning waste characterisation and land remediation. Measurement of ${ }^{3} \mathrm{H}$ activity concentrations in wastes is integral to the decommissioning of nuclear and military sites, fusion research and radiopharmaceutical manufacturing facilities and research laboratories. As ${ }^{3} \mathrm{H}$ is a low-energy pure beta-emitting radionuclide, in situ screening at low activities is not practically feasible and waste characterisation will typically involve the collection of a representative sub-sample followed by ex situ laboratory analysis. In order to obtain reliable data, it is vital that the sample is collected and stored in such a way as to prevent loss of ${ }^{3} \mathrm{H}$ prior to analysis and that the analytical approach adopted is capable of quantitatively extracting the form of ${ }^{3} \mathrm{H}$ present. To develop a robust sampling and analytical strategy the association of 
${ }^{3} \mathrm{H}$ in the sample must be well understood and this will depend on the origin of the ${ }^{3} \mathrm{H}$, the chemical form of ${ }^{3} \mathrm{H}$ within the sample and the sample composition. A review of our research into ${ }^{3} \mathrm{H}$ association in a range of commonly encountered sample matrices collected from different sites is presented. The implication of ${ }^{3} \mathrm{H}$ speciation and sample composition on analytical strategies is considered and experience in the application of these procedures to current decommissioning programmes in both the nuclear and radiopharmaceutical industries is also discussed.

\subsubsection{Analytical speciation of ${ }^{3} \mathrm{H}$ in environmental samples \\ (A. Bacchetta and N. Baglan)}

To determine tritium distribution in the environment, all fractions have to be measured and therefore to be separated. Usually, Tissue Free Water Tritium and $O B T$ are measured and the relation $T_{\text {total }}=T F W T+O B T$ (Ansoborlo et al., 2010) has already been proved through intercomparisons. However, $O B T$ is measured with no distinction between exchangeable and non-exchangeable $O B T$. The dry matter is immersed in water without tritium to promote an isotopic exchange between $E-O B T$ and hydrogen of water. However, at the end of the labile exchange, exchange waters are coloured. This coloration could arise from the solubilisation of organic molecules in water and thus disturb $O B T$ distribution. From this experimental evidence, two main research approaches are under investigation.

The first approach is to investigate variations in the hydrogen content in the sample during the labile exchange, which might carry a bias in $N E-O B T$ activities. Experimental results show different behaviours between samples and led to defining three classes, based on the hydrogen behaviour before and after exchange (Bacchetta and Baglan, 2013):

I) statistical equality of the mean and overlapping of confidence intervals,

II) statistical difference and overlapping of confidence intervals,

III) statistical difference and no overlapping of confidence intervals with, for the latter, potential bias in $N E-O B T$ quantification.

The second approach is to reach understanding of tritium behaviour in the environment by identifying compounds solubilised during the labile exchange. By using several analytical tools (elemental analyser, spectrophotometer, highresolution mass spectrometry), preliminary identifications which are consistent with the type of sample placed in exchange water were obtained, as carbohydrates for apple and monolignols for oak were found.

For a better understanding of tritium behaviour it is essential to identify more completely which molecules are soluble in water by coupling a separative technique with mass spectrometry. 


\subsubsection{New oxygen combustion bomb for low-level tritium assay}

(R. Marsh, I.W. Croudace, P.E. Warwick and M. McCarthy)

Nuclear facilities, particularly those conducting reprocessing operations, frequently release significant quantities of tritium and carbon-14 to the environment as part of authorised discharges. Analytical techniques for the determination of organically bound tritium $(O B T)$ in environmental biota typically rely on extraction via staged thermal desorption in a tube furnace system followed by measurement using liquid scintillation counting ( $L S C$ ). Thermal desorption is highly effective for most sample types; however, in the instance of matrices with a high organic content it is necessary to reduce the sample size considerably to prevent uncontrolled or incomplete combustion. This is a significant disadvantage when environmental samples are concerned, as a large sample size is typically required to ensure appropriate limits of detection are achieved. The Hyperbaric Oxidiser ( $H B O)$ (www.raddec.com) represents a new generation of oxygen combustion bomb designed by an $O B T$ research group in the UK specifically for the purpose of liberating tritium activity from large, organic-rich samples. Its use can contribute to the achievement of low limits of detection. The performance of this instrument has been evaluated through the combustion of standards containing both tritiated water and $O B T$, indicating that recoveries in excess of $98 \%$ are routinely achievable. In addition, a number of marine biota samples have been analysed on an intercomparison basis in collaboration with colleagues from the Institut de Radioprotection et de Sûreté Nucleairé (IRSN, Cherbourg-Octeville, France) to demonstrate the potential of the HBO for low-level environmental tritium assay.

\subsubsection{Variable OBT/HTO ratio in the aquatic environment (E. Fourré and P. Jean-Baptiste)}

$O B T / H T O$ ratios in terrestrial plants and food items are often substantially greater than one, even far away from local tritium sources: for samples representative of "background" environmental conditions, the mean $O B T / H T O$ value is close to 2 (Pointurier et al., 2004; Baglan et al., 2005), with measured values sometimes up to 3 or 4 (Jean-Baptiste et al., 2011). This tritium enrichment has often been attributed to soil moisture with presumably lower tritium content than atmospheric water vapour. More recently, it has been shown that the annual atmospheric water vapour tritium cycle, with its spring maximum which occurs during the growing season, is capable of explaining much of the observed terrestrial $O B T / H T O$ values (Jean-Baptiste et al., 2011).

According to these views, $O B T / H T O$ ratios in aquatic samples should not exhibit such enrichments. Most published $O B T / T F W T$ values for aquatic samples are indeed less than 1 (ASN, 2010). Nevertheless, our own measurements in both 
riverine and marine organisms collected over the last ten years suggest a more complex situation, with both "normal" and enriched $O B T / H T O$ values.

In fact, in natural environments, aquatic organisms grow in a mix of water and suspended matter. When analysed separately, this suspended matter often reveals higher tritium contents. Therefore, enriched $O B T / H T O$ ratios in aquatic organisms could be due to a suspended matter component, either retained at the surface of algae, for example, or ingested by fish or molluscs while feeding.

As most of the organic matter in sediments has a terrestrial origin (soil erosion of catchment basins, etc.), a better understanding of OBT/HTO ratios of organic matter in soils is necessary to interpret data from riverine and coastal samples. Carbon studies of soil organic matter (Balesdent and Recous, 1997) estimate the residence time of organic matter in soils at about 40 years. Tritium in soil organic matter originates from $H T O$ and bacterial degradation of $H T$. From (1) the history of atmospheric $H T$ and of $H T O$ in precipitation, and (2) estimates of the global $\mathrm{H}_{2}$ uptake by soil organic matter through bacterial activity, we roughly estimate the present-day contribution of these two tritium sources to soil OBT to be in the range of 50-100 TU.

\section{Short conclusion}

OBT analysis suffers from the lack of both common definition and a wide variety of samples to process. For these reasons, to analyse tritium forms different protocols are currently carried out. Thus, it is not straightforward to compare results arising from different laboratories. Therefore, validated analytical procedures, summarising the "know-how" of the laboratories performing OBT analysis, need to be published to further improve the performance of $O B T$ analysis.

\subsection{Inter-laboratory exercises}

In order to improve $O B T$ analysis, several exercises were organised in France and Canada between 2001 and 2010, mainly focusing on $O B T$ itself without any separation between $E-O B T$ and $N E-O B T$. During this period, the number of participants increased regularly with a focus on tritium and particularly $O B T$, eventually reaching 7 participants in Canada and 14 in France for the most recent exercises. During these exercises, the relationship described as "overall tritium activity $=T F W T+O B T$ " was assessed experimentally. Within experimental uncertainties, dehydration does not seem to disturb the sample. At the same time, the observed dispersion of the activity decreased, but remained nonetheless too high. This prompted us to stop our work to focus on promoting analytical 
procedures which could be used in all of the laboratories to give $O B T$ results based on a validated procedure.

In the various laboratories, differences in the analytical methods being used (e.g. drying protocols, washing the dried sample to remove exchangeable $O B T$, combustion methods and combustion water collection, LSC methods and materials) exist, that could potentially play a role in the observed dispersion in the inter-laboratory exercises. To investigate this point, an intercomparison that includes quantification of effects related to variations in procedures between different $O B T$ laboratories would provide a better basis for determining the causes of the disagreements in $O B T$ results and a basis for adopting a reference procedure. However, the organisation of such exercises is, on a national scale, limited in frequency due to the huge amount of work associated with the exercises and a limited population.

\subsubsection{Empirical insights and considerations for the OBT inter-laboratory comparison of environmental samples (S.B. Kim and J. Roche)}

$O B T$ is an important tritium species that can be measured in most environmental samples such as plants, animals and soils. Currently, $O B T$ is not routinely measured by environmental monitoring laboratories around the world. In the past, there have been differences in $O B T$ analysis results between environmental laboratories. One possible reason for the discrepancies may be the different analytical methods. Therefore, inter-laboratory $O B T$ comparisons within the environmental laboratories are important and would provide a good opportunity for adopting a reference $O B T$ analytical procedure. There are no certified reference materials (CRMs) for environmental samples. Thus, quality assurance $(Q A)$, or verification of the accuracy of the $O B T$ measurement, is not possible. Alternatively, quality control $(Q C)$, or verification of the precision of the $O B T$ measurement, can be achieved. The measurement of $O B T$ concentrations in environmental samples is longer and more complex than the measurement of HTO, and has a higher associated uncertainty. Also, only limited information is available on its measurement. Previously conducted $O B T$ inter-laboratory exercises in Canada suggested that a few special considerations such as analytical apparatus, target $O B T$ concentrations, exercise materials, measurement methods, sample size and volume, sample deliverable, and reporting results are required for the international $O B T$ intercomparison exercise. An international $O B T$ interlaboratory comparison exercise will improve $O B T$ measurement quality in environmental samples and increase understanding of $O B T$ behaviour in the environment. 


\subsubsection{A review of the French intercomparison for OBT determination (N. Baglan and E. Ansoborlo)}

The measurement of tritium in its various forms (mainly gas $(H T)$, water (HTO) or solid (hydrides), is an important key step for evaluating health and environmental risks, and finally, dose assessment. In vegetable or animal samples, tritium is often associated with the free water fraction, but may be included in the organic form as $O B T$. In this case, 2 forms exist: i) a fraction called exchangeable or labile (E-OBT) which is bound to oxygen and nitrogen atoms, and ii) a so-called nonexchangeable fraction (NE-OBT) bound to carbon atoms. The main technique for tritium analysis is liquid scintillation, which enables the measurement of concentrations down to about one Bq.L ${ }^{-1}$. The standards (AFNOR, ISO) published so far, although in France the writing of a standard on $O B T$ analysis is in progress, relate only to tritium analysis in water. Only one CETAMA method addresses $O B T$ analysis in samples originating from the environment. This method has been tested since 2001 through intercomparison circuits on grass samples collected from the environment. Regarding tritium analysis in water, the strengths are the reliability of this analysis at low concentrations (order of 1 Bq. $\mathrm{L}^{-1}$ ), robustness and simplicity, and the weaknesses are linked to problems of background, conservation and contamination of samples. Concerning $O B T$ analysis, the analysis is reliable for values around $50 \mathrm{~Bq} \cdot \mathrm{kg}^{-1}$ of fresh sample (Baglan et al., 2010). The weaknesses are problems of contamination, reproducibility, analysis time (2 to 6 days) and lack of reference materials. The key point today is the separation between E-OBT and NE-OBT that will need experimental validation in order to fully understand tritium behaviour within the environment.

\section{Short conclusion}

Work to promote the use of validated procedures is ongoing in several countries. However, for almost all interlaboratory exercise organisers it is difficult on a yearly basis to provide the samples and to carry out the statistical treatment of the results. Therefore, an international collaboration devoted to the improvement of OBT analytical procedures was proposed to overcome these limitations.

\section{Conclusions}

At the end of the workshop, the CEA and AECL proposed during the discussion to create an international working group devoted to $O B T$ analysis. The participants were for the most part very interested in this, especially since in most countries where tritium discharges occur, $O B T$ might eventually become a regulatory measurement. Therefore, it was decided to create a task group led by both AECL 
and the CEA with the support of the scientific committee of the workshop. The main goal of this group will be to promote $O B T$ analysis through inter-laboratory exercises over a three-year period ending in 2015. To date, 35 laboratories from about 9 countries have expressed interest in participating in this group. For the first intercomparison, the samples will be provided in December 2012 by AECL, who will produce enough specially-prepared potatoes to allow each participant to realise the five replicates necessary to allow a reliable statistical treatment. The following exercises will be organised using sediment and animal samples. The expected benefits of this are the following: remove or reduce uncertainty in $O B T$ analysis results, provide better $O B T$ model validation data and better public dose results, increase the number of potential $O B T$ laboratories, and validate all of the stages of the procedures based on a larger population and more statistically significant results. At the end of the three-year period, depending on the conclusions of the first period, the exercises might continue, either to validate the analytical procedure or as routine exercises.

\section{REFERENCES}

AGIR (2007) Advisory Group on Ionising Radiation, Review of risks from tritium documents of the health Protection agency: Radiation, chemical and Environmental Hazards, REC 4, November 2007. Available from http://www.hpa.org.uk/publications.

Ansoborlo A. et al. (2010) Circuit intercomparaison 2009-2012, tritium organiquement lié dans une matrice herbe, Note Technique DEN/DCRP/CETAMA/NT/2010/03.

ASN (2010) Livre Blanc du Tritium, Autorité de Sûreté Nucléaire, Available from http://www.asn.fr.

Bacchetta A., Baglan N. (2013) Labile exchange impact on NE-OBT quantification (in preparation).

Balesdent J., Recous S. (1997) Le temps de résidence du carbone et le potentiel de stockage de carbone dans quelques sols cultivés français, Can. J. Soil. Sci. 77, 187-193.

Baglan N., Alanic G., Pointurier F. (2005) Tritium determination at trace level. Which strategy to determine accurately HTO and OBT in environmental samples? Fusion Sci. Technol. 48, 749754.

Baglan N., Ansoborlo E., Cossonnet C., Fouhal L., Deniau I., Mokili M., Henry A., Fourré E., Olivier A. (2010) Métrologie du tritium dans différentes matrices: cas du tritium organiquement lié, Radioprotection 45 (3), 369-390.

CNSC (2010) Canadian Nuclear Safety Commission, Health Effects, Dosimetry and Radiological Protection of Tritium, Info-0799.

Croudace I.W., Warwick P.E., Morris J.E. (2012) Evidence of the preservation of technogenic tritiated organic compounds in an estuarine sedimentary environment, Environ. Sci. Technol. 46, 57045712.

ICRP Publication 72 (1995) Age-dependent Doses to Members of the Public from Intake of Radionuclides, Ann. ICRP 26 (1).

Jean-Baptiste P., Fourré E., Baumier D., Dapoigny A. (2011) Environmental OBT/TFWT revisited, Fusion Sci. Technol. 60, 1248-1251.

LNE (2006) LNE-LNHB/CEA, Table de radionucléides.

Pointurier F., Baglan N., Alanic G. (2004) A method for the determination of low level organic-bound tritium activities in environmental samples, Appl. Radiat. Isotopes 61, 293-298. 